\title{
Hybrid Energy Efficiency Friendly Frequency Domain TR Algorithm Based on PSO Algorithm Evaluated by Novel Maximizing HPA Efficiency Evaluation Criteria
}

\author{
Feng Hu ${ }^{1,+}$, Yuan Lu ${ }^{1, *,+}$, Libiao Jin ${ }^{1,+}$, Jianbo Liu ${ }^{1,+}{ }^{+}$Zhiping Xia ${ }^{2}$, Guoting Zhang ${ }^{2}$ and Jingting Xiao ${ }^{2}$ \\ 1 School of Information and Communication Engineering, Communication University of China, \\ Beijing 100024, China; fenghu@cuc.edu.cn (F.H.); libiao@cuc.edu.cn (L.J.); ljb@cuc.edu.cn (J.L.) \\ 2 Academy of Broadcasting Science, Beijing 100045, China; xiazhiping@abs.ac.cn (Z.X.); \\ zhangguoting@abs.ac.cn (G.Z.); xiaojingting@abs.ac.cn (J.X.) \\ * Correspondence: luyuancuc@cuc.edu.cn \\ + These authors contributed equally to this work.
}

check for updates

Citation: Hu, F.; Lu, Y.; Jin, L.; Liu, J.; Xia, Z.; Zhang, G.; Xiao, J. Hybrid Energy Efficiency Friendly Frequency Domain TR Algorithm Based on PSO Algorithm Evaluated by Novel Maximizing HPA Efficiency Evaluation Criteria. Energies 2022, 15, 917. https://doi.org/10.3390/ en15030917

Academic Editors: William Holderbaum and Mauro Feliziani

Received: 26 November 2021

Accepted: 26 January 2022

Published: 27 January 2022

Publisher's Note: MDPI stays neutral with regard to jurisdictional claims in published maps and institutional affiliations.

Copyright: (C) 2022 by the authors. Licensee MDPI, Basel, Switzerland. This article is an open access article distributed under the terms and conditions of the Creative Commons Attribution (CC BY) license (https:// creativecommons.org/licenses/by/ $4.0 /)$.

\begin{abstract}
Smart Grids (SGs) expedite secure, large-scale and efficient two-way communication between the power supply and management, but under a sophisticated 5G communication infrastructure, the multi carrier system is the principle system. The high peak-to-average power ratio (PAPR) is one of the significant limitations of the $5 \mathrm{G}$ multi carrier (MC) system, as it impedes the efficient design of the $5 \mathrm{G}$ analogue front end. Tone reservation (TR) is a highly efficient scheme without signal distortion, which is designed by increasing the freedom in the frequency domain for PAPR reduction. In this paper, a particle swarm optimization (PSO) based TR (PSO-TR) scheme proceeding with an optimal input power back off-modulation error ratio (IBO-MER) convergence criterion is proposed to improve high power amplifier (HPA) efficiency for OFDM systems. A probabilistic analysis of TR predistribution and freedom in the frequency domain, in relationship with the amplitude of its constituent samples, is carried out. This yields the theoretical framework employed in design of the proposed high computing-enhanced solutions. The proposed PSO-TR essentially make the frequency domain distribution and operation itself adaptive, that is, it adjusts to comply with the changing HPA efficiency and redundant cost during application runtime.
\end{abstract}

Keywords: OFDM; PAPR; HPA; TR; PSO; 5G; PSO; IBO; MER; energy efficiency

\section{Introduction}

The 5th communication system supports a wide range of wireless services by applying a millimeter-Wave and multicarrier modulated system, which significantly improves data rate and time delay [1]. However, the demand for higher carrier frequency and broader bandwidth with limited energy efficiency sacrifice is the major challenge in 5G communication systems and SGs [2]. The power amplifier (PA) is the most power-consuming device among traditional transmission components in general, which takes $50 \% \sim 80 \%$ of total power consumption from a base station [3]. Therefore, HPA improvement is significant for enhancing the energy efficiency of the base station.

Orthogonal frequency division multiplexing (OFDM) has been widely chosen for 5G wireless communication standards due to many desirable properties such as high spectral efficiency, simple channel estimation, and robustness against multipath fading [4]. The major drawback of OFDM is the high peak-to-average power ratio (PAPR) characteristic caused by the superposition of peaks from a large number of subcarriers [5]. High PAPR can drive the signal beyond the linear region of the HPA, which can cause serious signal distortion [6], modulation error rate (MER) decreases, demodulation errors and deterioration of PA efficiency. The minimum (complementary cumulative distribution function) CCDF curve is the most common criterion to evaluate PAPR method function [7]. 
However, due to the low probability of peak value appearance, CCDF improvement does not necessarily imply optimising PA efficiency. Signal peak can be optimization with a variety of approaches and schemes: clipping and filtering (CF) [8]; coding [9]; adaptive symbol selection [10]; partial transmit sequence (PTS) [11] and interleaving [12]; tone reservation/injection [13]; active signal constellation extension (ACE) [14], companding, and so forth [15]. Predistortion and coding schemes are no longer sufficient for modern communication systems; he tone distortion algorithm is considered one of the most potential approaches. TR is a commonly used PAPR reduction technique in 5G communication systems; many optimization TR algorithms have been proposed to obtain a global optimized PAPR reduction result. Refs. [16-18] combined PTS, ACE, and clipping schemes with TR, PARP reduction performance has been improved at the cost of increased computational complexity and SNR. Refs. [19,20] applied machine learning (PRNet, deep neural network) with the TR scheme which can minimize PAPR by training examples; a lack of training data and extensive training time is the main concern of such algorithms.

By increasing the freedom of a signal in the frequency domain space and its space, signals in frequency domain QAM coordinates can expand freely within an allowable range, which acquires a higher level of freedom than conventional multi carrier systems, so that the probability of the phase superposition of high peak signal can be reduced, and PAPR can be reduced effectively [21]. Therefore, the TR distortion process can be advanced to the frequency domain as F-TR. To describe and optimize the distortion of HPA, we proposed a novel maximizing HPA efficiency evaluation criteria IBO-MER to statistically analyze the amplitude and occurrence probability of the signal level, balance considered distortion influence from each subcarrier, and obtain the best CCDF curve distribution of OFDM signal. To achieve the dual optimization targets of HPA energy consumption reduction and system energy efficiency improvement and reach a compromise between signal freedom and HPA power amplifier efficiency, we proposed an optimal particle swarm optimization based (PSO) algorithm to analyze the global boundary. By PSO algorithm, global maximum/minimum optimization objectives can be searched, the global boundary of the objective function can be determined [22]. Specifically, we proposed a novel scheme based on the continuous PSO genetic algorithm to achieve the best OFDM signal distribution. FPSO-TR can reach theoretical boundaries in the continuous infinity domain. Compared with conventional TR, FPSO-TR has reached optimal PAPR reduction and MER results within reasonable complexity.

This paper is arranged as follows: descriptions of MIMO-OFDM and its inherent PAPR characteristics are presented in Sections 2.1 and 2.2. The HPA model and its characteristics are described in detail in Sections 2.3 and 2.4. In Section 3, the conventional TR scheme is shown. Analytical evaluations of the F-TR and FPSO-TR algorithms are contained in Section 4. Finally, simulations and concluding remarks are given in the last section.

\section{Massive MIMO-OFDM Model}

\subsection{MIMO-OFDM Model}

In this section, we present the MIMO-OFDM system and its PAPR characteristic. The continuous-time domain OFDM with $N$ subcarriers could be expressed as [23]:

$$
x(t)=\sum_{k=0}^{N-1} X_{k}(t) e^{j 2 \pi k \Delta f t}, 0 \leq t \leq T,
$$

where $X(k)$ is OFDM complex signals in $k$-th subcarrier, $T$ is signal duration, OFDM signals are modulated with 16 QAM in this paper.

Similarly, the discrete-time domain OFDM signals can be represented as [23]:

$$
x(n)=\frac{1}{\sqrt{N L}} \sum_{k=0}^{N L-1} X_{k}(n) e^{j 2 \pi f_{k} n / L N}, n=0,1, \ldots, N L-1,
$$


where $L$ is the oversample times.

An OFDM-MIMO system composed of a base station (BS) with $M$ transmitter (TX) and receiver (RX) antennas has $Q$ users. After inverse discrete Fourier transform (IDFT), the frequency domain signal $\mathbf{Y}[k]=\left[Y_{0}[k], Y_{1}[k], \ldots, Y_{M-1}[k]\right]^{\mathrm{T}}$ of $k$-th subcarrier can be represented as [24]:

$$
\mathbf{Y}[\mathbf{k}]=\sqrt{\rho} \mathbf{H}[k] \mathbf{X}[k]+\mathbf{N}[k], 0 \leq k \leq N-1
$$

where $\mathbf{Y}[\mathbf{k}]$ is $M \times 1$ received vector, $\rho$ is the total TX power for a downlink signal, $\mathbf{H}[\mathbf{k}]$ denotes the $M \times M$ independent identically distributed channel matrix between TX and $R X$, $\mathrm{X}[\mathbf{k}]$ represents the $M \times 1$ signal vector with each data symbol modulated by quadrature amplitude modulation (QAM), and $\mathbf{N}[\mathbf{k}]$ represents the $M \times 1$ additive white Gaussian distribution.

The discrete-time $t$-th OFDM-MIMO signal of TX antenna $x_{t}=\left[x_{t}[0], x_{t}[1], \ldots, x_{t}[N-1]\right]^{T}$ can be represented as [25]:

$$
\mathbf{x}_{t}=\mathbf{F}_{q}^{\mathrm{H}} \mathbf{X}_{t}
$$

where $\mathbf{X}_{t}=\left[X_{t}[0], X_{t}[1], \ldots, X_{t}[N-1]\right]^{\mathrm{T}}$ is the frequency domain signal after IDFT, $\mathbf{F}_{q}^{\mathrm{H}} \in$ $\mathbb{C}^{N q \times N}$ represents IDFT matrix as $\left[\mathbf{F}_{q}\right]_{i, k}=\frac{1}{\sqrt{q} N} e^{-j 2 \pi i k / q N}$ with its $(i, k)$-th element.

\subsection{PAPR Definition}

The definition of PAPR is the ratio of the peak and average power of the multi carrier system, which is the character of the signal peak's superposition caused by amplitude Rayleigh distribution. PAPR can be represented as [26]:

$$
\text { PAPR }=\frac{\max _{0 \leq n \leq q N-1}\left\{\left|\mathbf{x}_{t}[n]\right|^{2}\right\}}{\mathrm{E}\left[\left|\mathbf{x}_{t}[n]\right|^{2}\right]},
$$

where $E\{\cdot\}$ is the expectation operator.

Complementary Cumulative Distribution Function (CCDF) is the primary evaluation criterion of PAPR in OFDM signals only to seek for the minimize PAPR value, which counts the probability that the PAPR of OFDM symbol $x_{t}[n]$ exceeds a particular threshold value $Z_{0}$, which can be shown as [27]:

$$
\begin{aligned}
& \operatorname{CCDF}_{P A P R}\left(\text { PAPR }_{0}\right)=\operatorname{Pr}\left(\operatorname{PAPR}>\text { PAPR }_{0}\right) \\
& =1-\mathrm{F}(Z) \\
& =1-\left(1-\mathrm{e}^{-Z_{0}}\right)^{N} .
\end{aligned}
$$

CCDF measures a sparse clipping probability, which represents the high peak sample is likely to undergo severe distortion but ignores the information on how many samples are distorted [27]. Moreover, the secondary peaks with considerable statistical quantity are more important in evaluating the HPA energy efficiency. Indeed, CCDF alone cannot predetermine the optimal target of each sub-carrier and entire distribution in HPA. In practical scenarios, it is essential to consider a more refined analysis considering all the signal samples.

\subsection{HPA Model}

Excessive PAPR would force HPA operation in the saturated region. To evaluate OFDM transmitter system, it's crucial to represent HPA models. The discrete signal $x_{t}[n]$ can represented as:

$$
x_{t}[n]=\left|x_{t}[n]\right| e^{j y_{t}[n]},
$$


where $\left|x_{t}[n]\right|$ and $e^{j y_{t}[n]}$ is the amplitude phase of signal $x_{t}[n]$, respectively. Amplified signal $y_{t}[n]$ can be represented as follows:

$$
y_{t}[n]=A\left[\left|x_{t}[n]\right|\right] e^{j\left\{y_{t}[n]+\phi\left[\left|x_{t}[n]\right|\right]\right\}},
$$

where $A[\cdot]$ and $\phi[\cdot]$ is Amplitude/Amplitude (AM/AM) and Amplitude/Phase (AM/PM) conversion, respectively.

This paper adopted a nonlinear Rapp model without memory for the HPA. The Rapp model for a solid-state power amplifier (SSPA) was developed in [28], which is a memoryless HPA model with only an AM/AM function. The Rapp model can be represented as:

$$
\begin{aligned}
& \tilde{\mathbf{x}}_{t}[n]=\frac{\sqrt{G} \mathbf{x}_{t}[n]}{\left(1+\left(\frac{\mathbf{x}_{t}[n]}{A_{s a t}}\right)^{2 p}\right)^{\frac{1}{2 p}}} \\
& \phi[|x[n]|] \approx 0,
\end{aligned}
$$

where $A_{\text {sat }}$ is the amplifier saturation voltage, $p$ is the parameter that controls the AM/AM sharpness of the saturation region, and $G$ is the amplifier voltage gain, considered as unitary in this paper for simplicity.

It is known that the characteristic of $\mathrm{AM} / \mathrm{AM}$ is more benign than that of $\mathrm{AM} / \mathrm{PM}$ when high order modulations are employed [29].

\subsection{Power Consumption Model}

In practical communication systems, the total power consumption contains the power radiated to the environment for signal transmission and an independent transmission power representing the power consumed by circuit dissipation and signal processing. We suppose that the constant power is proportional to the transmit antenna number, then the realistic total power consumption is given as [30]:

$$
P_{\text {total }, \ell}=\sum_{a \in \ell} \frac{P_{a}}{\eta_{a}}+\ell \cdot P_{\text {cir }}+P_{\text {sta }},
$$

where $\eta_{a}$ denotes the PA efficiency for the $a$-th antenna, $P_{a}$ is the maximal HPA output power for the $a$-th transmit antenna, $\ell$ denotes the active transmit antenna set of the BS, and $\ell \subseteq\{1, \ldots, M\} . \ell \cdot P_{\text {cir }}$ denotes the circuit power consumption proportional to the number of the active transmit antennas. $P_{s t a}$ denotes the static power independent of both $P_{a}$ and $\ell$, which accounts for the power consumption of the baseband processing, and so forth.

\subsection{Performance Metrics}

The power amplifier's distortion can be classified as nonlinear distortion (amplitude distortion) and cut-off saturation distortion (frequency and phase distortion). To optimize the dynamic range of the power amplifiers, he signal power from the transmitter of HPA can be backed off.

The input back-off (IBO) is defined as the operating point of the HPA, which can be represented as [31]:

$$
I B O=10 \log _{10} \frac{A_{\text {sat }}^{2}}{P_{\text {avg }}}=10 \log _{10} \gamma^{2}, \gamma=\frac{A_{\text {sat }}}{\sqrt{P_{a v g}}},
$$

where $P_{\text {sat }}$ denotes the average input power.

It has been well investigated that the OFDM signal converges to a complex Gaussian random process when $N$ is large enough according to the central limit theorem. For the Gaussian input $x_{t}(n)$, the Bussgang theorem can be employed. Alternatively, the output signal of the HPA $\overline{\mathbf{x}}_{t}[n]$ can be disassembled into two uncorrelated signals based on 
Bussgang theorem, that is, $\overline{\mathbf{x}}_{t}[n]=\alpha \mathbf{x}_{t}[n]+\mathbf{d}_{t}[n]$ where a represents an attenuation factor and the distortion term $\mathbf{d}_{t}[n]$ is uncorrelated with xi(n) $\left(\mathbf{d}_{t}[n]\right.$ and $\left.\mathrm{E}\left[\mathbf{d}_{t}[n] \mathbf{x}_{t}^{*}[n]\right]=0\right)$. The attenuation factor a is depend on the IBO level is as follows:

$$
\alpha=\frac{\mathrm{E}\left(\mathbf{x}_{t}^{*} 25[n] \overline{\mathbf{x}}_{t}[n]\right)}{\mathrm{E}\left[\left|\mathbf{x}_{t}[n]\right|^{2}\right]}=1-\mathrm{e}^{-\gamma}+\frac{\sqrt{\pi} \gamma}{2} \operatorname{erfc}(\gamma)
$$

where $\operatorname{erfc}(\gamma)=\frac{2}{\sqrt{\pi}} \int_{\gamma}^{\infty} e^{-x^{2}} d x$ is the error function.

The distortion induced by the Rapp function $(p \rightarrow \infty)$ impacts the tail of the Rayleigh distribution. Hence, the variance of $d_{t}[n]$ can be easily obtained as follows:

$$
\begin{aligned}
\sigma_{d}^{2} & =\mathrm{E}\left[\left|\overline{\mathbf{x}}_{t}[n]\right|^{2}\right]-\alpha^{2} \mathrm{E}\left[\left|\mathbf{x}_{t}[n]\right|^{2}\right] \\
& =\left(1-\mathrm{e}^{-\gamma^{2}}-\alpha^{2}\right) \sigma^{2}
\end{aligned}
$$

where $\left(1-\mathrm{e}^{-\gamma^{2}}\right) \sigma^{2}$ represents the variance of $\overline{\mathbf{x}}_{t}[n]$.

In this paper, the signal-to-distortion ratio (SDR) is due to distortion noise caused by the HPA, which can be defined as follows [32]:

$$
\begin{aligned}
\operatorname{SDR}(\hat{\mathbf{x}}) & =10 \lg \left\{\frac{\alpha^{2} \mathrm{E}\left[\left|\mathbf{x}_{t}[n]\right|^{2}\right]}{\left.\mathrm{E}\left[\left|\overline{\mathbf{x}}_{t}[n]-\mathbf{x}_{t}[n]\right|^{2}\right]\right\}}\right\} \\
& =10 \lg \frac{\alpha^{2} \sigma^{2}}{\sigma_{d}^{2}} \\
& =10 \lg \frac{\alpha^{2}}{\left(1-\mathrm{e}^{\left.-\gamma^{2}-\alpha^{2}\right)^{\prime}}\right.}
\end{aligned}
$$

where $\left[E\left[\left|\mathbf{x}_{n}\right|^{2}\right]=\sigma^{2}\right.$ is the power of the input signal. The SDR is relevant to the reception quality. Most importantly, the utilized definition is a wideband SDR, that is, covering both in-band and out-of-band frequencies. Notice that SDR depends on the IBO level $\gamma^{2}$, and the attenuation $\alpha$. Signal power is calculated only for data symbols.

Distortion power is sparse and distributed in the time domain, uniform in the frequency domain receiver. HPA distorted symbols in the frequency domain can be shown as:

$$
\tilde{\mathbf{Y}}[k]=\left[\tilde{Y}_{0}[k], \tilde{Y}_{1}[k], \ldots, \tilde{Y}_{M-1}[k]\right]^{\mathrm{T}},
$$

where

$$
\begin{aligned}
& \tilde{\mathbf{Y}}[k]=\sqrt{\rho} \mathbf{H}[k] \overline{\mathbf{X}}[k]+\mathbf{N}[k] \\
& =\sqrt{\rho} \mathbf{H}[k](\alpha \mathbf{X}[k]+\mathbf{D}[k])+\mathbf{N}[k]
\end{aligned}
$$

and $D[k]=\left[D_{0}[k], D_{1}[k], \ldots, D_{M-1}[k]\right]^{\mathrm{T}}$ represents the frequency domain version of the nonlinear distortion terms.

Energy efficiency (EE) is one of the key metrics to evaluate HPA performance, which is defined as the number of information bits transmitted per unit of energy. Assuming that IBO is appropriately set, and distortion due to the nonlinear devices is negligible, the EE can be represented as [30]: 


$$
\begin{aligned}
& E E \approx \frac{\kappa \cdot \mathrm{E}\left[\log _{2} \operatorname{det}\left(\mathbf{I}_{\mathrm{M}}+\frac{\rho \mathbf{H}[k] \mathbf{H}^{H}[k]}{\ell N_{0} B}\right)\right]}{P_{\text {total }, \ell}} \\
& \text { s.t } \xi \leq \vartheta, \ell \leq M,
\end{aligned}
$$

where $\mathbf{I}_{\mathrm{M}}$ is the $M \times M$ identity matrix, $N_{d} B$ is the noise power at a given bandwidth, $B . \kappa$ is the scaling factor for the signaling overhead and guard interval, which can be shown as:

$$
\kappa=(1-\xi),
$$

where $\kappa$ represents pilot ratio.

HPA efficiency $\eta_{a}$ is usually defined to measure the EE based on HPA operation. High $\eta_{a}$ offers low power consumption, in an ideal HPA model, $\eta_{a}$ is modelled by a constant, and the total power consumption is a linear function of the radiated power of the HPA. However, $\eta_{a}$ has a dependence on the radiated power HPA in practical systems. According to [33], a more accurate HPA model can be described as follows:

$$
\eta_{a}=\left(\frac{P_{a}}{P_{a, \max }}\right)^{\beta} \eta_{\max }
$$

where $P_{a}$ is the maximal HPA output power for the $a$-th transmit antenna, $\eta_{\max }$ is the maximal HPA efficiency. $\beta$ is the efficiency exponent depending on the type of the HPA where $\beta \in[0,1]$. For class B and class A non-ideal HPAs, the typical value of $\beta$ is 0.5 and 1 , respectively. From (14), it has known that the efficiency of HPA decreases with the decline of HPA output power.

Modulation error rate (MER) is a significant criterion for measuring signal quality by quantifying signal to noise ratio (SDR), defined as the ratio of the received symbol location and the difference between received symbol location and ideal one. HPA efficiency and distortion have a tradeoff relationship, as mentioned previously. The lower IBO leads to higher output signal power and, thus, higher HPA efficiency, but it may also cause severe nonlinear distortion. The in-band SDR contribution in (11) can be calculated by computing the quantity MER, which can be shown as follows [34]:

$$
\begin{aligned}
\operatorname{SDR}(\hat{\mathbf{x}}) & \approx \operatorname{MER}\left(\widehat{\mathbf{S}}_{\mathbf{n}}\right) \\
& =10 \lg \left\{\frac{\alpha^{2} \sum_{k=1}^{N}\left(\left|s_{k}\right|^{2}\right)}{\sum_{k=1}^{N}\left(\left|s_{k}-\widehat{s}_{k}\right|^{2}\right)}\right\} \\
& =10 \lg \left\{\frac{\alpha^{2} \sum_{k=1}^{N}\left(I_{k}^{2}+Q_{k}^{2}\right)}{\sum_{k=1}^{N}\left(\Delta I_{k}^{2}+\Delta Q_{k}^{2}\right)}\right\},
\end{aligned}
$$

where $N$ is the number of data samples, $I$ and $Q$ are the real and imaginary ideal locations, $\Delta I$ and $\Delta Q$ are the real and imaginary difference between received symbol locations and the ideal one. MER can indirectly evaluate the performance of OFDM acceptance.

\section{Conventional TR System}

In single-antenna OFDM systems, the TR-based scheme reserves $N_{R}$ peak reduction tones (PRTs) set for PAPR reduction and rest $N-N_{R}$ subcarriers for data transmission. The time domain kernel $c=\left[c_{0}, c_{1}, \cdots, c_{N q_{-1}}\right]^{\mathrm{T}}$ is generated as the peak-canceling signal by the $R$ reserved tones, which can be calculated as [13]: 


$$
\mathrm{c}=\mathrm{FC},
$$

where $\mathbf{F}$ represents the inverse fast Fourier transform matrix, $\mathbf{C}=\left[C_{0}, C_{1}, \cdots, C_{N-1}\right]^{\mathrm{T}}$ represents the reserved frequency domain kernel with nonzero tones at positions in $R$, which can be shown as:

$$
C_{k}=\left\{\begin{array}{l}
0, k \notin \mathbf{R} \\
1, k \in \mathbf{R},
\end{array}\right.
$$

where $R$ is the number of reserved tones, and $\mathbf{R}=\left[r_{0}, r_{1}, \cdots, r_{R-1}\right]^{\mathrm{T}}$ denotes the set of the reserved tones.

So that the time domain signal in (2) after TR peak cancelling transfer into:

$$
\mathbf{a}=\mathbf{x}+\mathbf{c}=\mathrm{F}(\mathbf{X}+\mathbf{C}),
$$

where $\mathbf{a}$ is the peak reduced time domain signal, and $\mathbf{X}$ and $\mathbf{C}$ are orthogonal in the frequency domain. To be specific, the frequency domain $n$-th subcarrier peak-cancelling signal can be represented as:

$$
X_{n}+C_{n}=\left\{\begin{array}{l}
X_{n}, n \notin \mathbf{R} \\
C_{n}, n \in \mathbf{R} .
\end{array}\right.
$$

The structure of the conventional TR scheme is given in Figure 1.

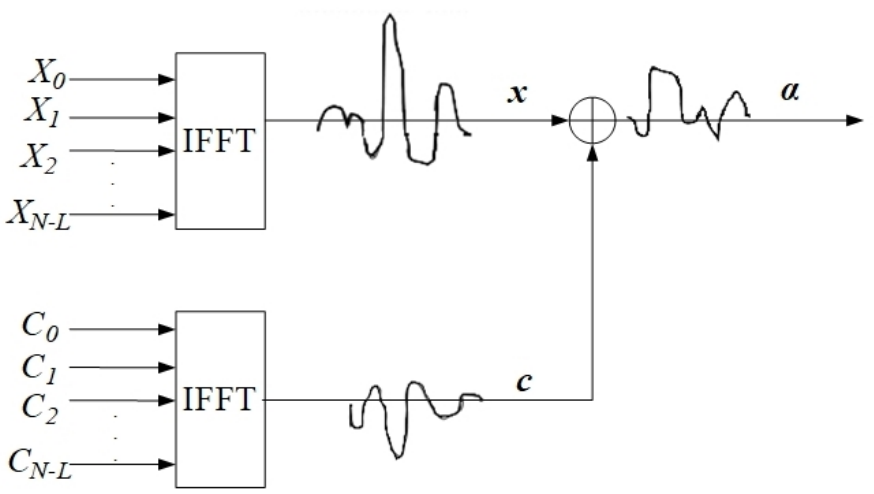

Figure 1. The structure of the conventional TR scheme.

In MIMO-OFDM systems, each antenna transmits a modulated OFDM signal. To avoid tones' contamination of the MIMO receiver, the same $\mathbf{C}=\left[C_{0}, C_{1}, \cdots, C_{N-1}\right]^{\mathrm{T}}$ can be utilized to generate the peak-canceling signal on the $m$-th transmit antenna. Therefore, the TR scheme can reduce the PAPR of MIMO-OFDM systems by performing the TR iterations independently on every transmit antenna. Then, the PAPR reduced signal $\hat{\mathbf{x}}_{t}$ of $t$-th antenna is generated by the peak-cancelling signal c plus the original signal $\mathbf{x}_{t}$ in the time domain, which can be shown as:

$$
\hat{\mathbf{x}}_{t}=\mathbf{x}_{t}+\mathbf{c}=\mathbf{F}_{q}^{\mathrm{H}}\left(\mathbf{X}_{t}+\mathbf{C}\right) .
$$

To further improve the dispersion of the signal, the PAPR signal $\hat{\mathbf{x}}_{t}^{i}$ is updated during the $i$-th iteration as follows:

$$
\begin{aligned}
\hat{\mathbf{x}}_{t}^{i+1} & =\hat{\mathbf{x}}_{t}^{i}+\sum_{l=0}^{L-1} \partial_{l}\left(A_{i} e^{j \arg \left(x_{n_{l}}^{i}\right)}-x_{n_{l}}^{i}\right) \mathbf{c}\left[\left(n-n_{l}\right)\right] \\
& =\hat{\mathbf{x}}_{t}^{i}+\sum_{l=0}^{L-1} a_{l} \mathbf{c}_{l}[(n),]
\end{aligned}
$$

where $\partial_{l}$ is a scaling factor, and $\mathbf{c}\left(n-n_{l}\right)$ is the cyclically shifted of $c$ to $n_{l}, n_{l}$ denotes the position of whose value exceeds the amplitude $A_{i}$ in the $i$-th iteration, and $L$ is the number 
of the positions. The time-domain OFDM symbol of the $t$-th antenna $x_{t}$ to be transmitted can be represented as follows:

$$
\begin{aligned}
\hat{\mathbf{x}}_{t} & =\mathbf{x}_{t}+\sum_{i=1}^{M} \sum_{l=0}^{L-1} \mathbf{c}_{i, l} \\
& =\mathbf{F}_{q}\left(\mathbf{X}_{t}+\mathbf{C}_{t}\right),
\end{aligned}
$$

where $\mathbf{c}_{i}$ is the updated time domain kernel during the $i$-th iteration, and $M$ is the adaptive iteration number, and $\mathbf{C}_{t}$ represents the frequency domain kernel of $t$-th antenna.

The PAPR of TR-OFDM signals can be transferred into:

$$
\text { PAPR }=\frac{\max _{0 \leq n \leq q N-1}\left\{\left|\mathbf{x}_{t}[n]+c_{t}[n]\right|^{2}\right\}}{\mathrm{E}\left[\left|\mathbf{x}_{t}[n]\right|^{2}\right]} .
$$

Moreover, the optimum PAPR reducing performance can be selected as follows:

$$
\begin{aligned}
& \arg \underset{\mathbf{C}_{t}}{\min } \max \mathbf{F}_{q}\left(\mathbf{X}_{t}+\mathbf{C}_{t}\right)_{\infty} \\
& \text { s.t. } \sum_{i=0}^{N-1} C_{t, i^{\prime}}^{2}, \leq \beta
\end{aligned}
$$

where $\beta$ is the maximum allowed power increasing imposed on reserved tones to avoid dramatically increasing the transmit power caused by reserved tones superposition.

The essential algorithm of conventional TR is exhaustively searching in the time domain kernel to find the minimal secondary peaks at the expense of high computational complexity and time delay. Meanwhile, TR has an unsatisfactory performance when a high power peak is unlikely to appear because TR cannot distinguish the maximum peak in this scenario.

\section{Proposed Frequency-Particle Swarm Optimization-Tone Reservation Scheme with Novel Maximizing HPA Efficiency Evaluation Criteria}

This section proposed a novel FPSO-TR algorithm, saving much IFFT progress by conducting TR in the frequency domain. Instead of utilizing iteration to an exhaustive search for the suboptimal peak-cancelling kernel, this paper obtained the global optimal kernel by employing the PSO algorithm. To further statistically analyze the amplitude and occurrence probability of signal level, novel maximizing HPA efficiency evaluation criteria were applied and the effect of each subcarrier on the distortion of the power amplifier was taken into account.

\subsection{F-TR and Maximizing HPA Efficiency Evaluation Criteria}

By advancing TR into the frequency domain, F-TR can be achieved by severe clipping and frequency filtering so that the move of the correcting signal lies only on the reserved tones.

The frequency domain OFDM signal divided onto $N-L$ data transmitting signal $X_{t}=\left[X_{t}[0], X_{t}[1], \ldots, X_{t}[N-L]\right]^{T}$ and $L$ reserved tones $\mathbf{C}=\left[C_{0}, C_{1}, \cdots, C_{N-1}\right]^{\mathrm{T}}$. Then the PAPR reduced signal $x_{t}$ of $t$-th antenna is generated by the peak-cancelling signal $c$ plus the original signal $x_{t}$ in the time domain, which can be shown as:

$$
\begin{aligned}
\hat{\mathbf{x}}_{t} & =\mathbf{x}_{t}+\sum_{i=1}^{M} \sum_{l=0}^{L-1} \mathbf{c}_{i, l} \\
& =\mathbf{F}_{q}\left(\mathbf{X}_{t}+\mathbf{C}_{t}\right) .
\end{aligned}
$$


Then the time domain signal $\hat{\mathbf{x}}_{t}[n]=\left[\hat{\mathbf{x}}_{t}[0], \hat{\mathbf{x}}_{t}[1], \ldots, \hat{\mathbf{x}}_{t}[N-L]\right]$ was clipped to threshold Asat as:

$$
\bar{x}_{t}[n]=\left\{\begin{array}{cc}
\hat{\mathbf{x}}_{t}[n], & \text { if }\left|\hat{\mathbf{x}}_{t}[n]\right|<\mathrm{A}_{\text {sat }} \\
A_{s a t} e^{j \theta[n]}, & \text { if }\left|\hat{\mathbf{x}}_{t}[n]\right| \geq \mathrm{A}_{\text {sat }},
\end{array}\right.
$$

where $\bar{x}_{t}[n], n=0,1, \ldots, N-1$ is the clipped signal, $\theta[n]$ is the phase of $\bar{x}_{t}[n]$ whose amplitude exceeds $A_{\text {sat }}$.

Evidently, the clipping noise $\mathbf{w}(\mathbf{n}), \mathrm{n}=0,1, \ldots, \mathrm{N}-1$ can be obtained as:

$$
\mathbf{w}(\mathbf{n})=\mathbf{x}_{t}[n]-\overline{\mathbf{x}}_{t}[n] .
$$

Transfer clipping noise $\mathbf{w}$ into the frequency domain as:

$$
\mathbf{W}=\mathbf{F}_{q}(\mathbf{w}),
$$

where $\mathbf{w}=\left[w_{0}, w_{1}, \ldots, w_{q N-1}\right]$ is the the corresponding frequency domain clipping noise from $\mathbf{W}=\left[W_{0}, W_{1}, \ldots, W_{q N-1}\right]$, respectively.

To empty the data transmission subcarriers, reserve values of PRTs only, the frequency filtering $\mathbf{H}_{r}(k)$ were set as:

$$
\begin{array}{r}
\mathbf{C}(k)=\mathbf{W}(k) \mathbf{H}_{r}(k)=\left\{\begin{array}{cc}
\mathbf{W}(k), & k \in R \\
0, & \text { otherwise }
\end{array}\right. \\
\underset{0 \leq k \leq q N-1}{\mathbf{H}_{r}(k)}=\left\{\begin{array}{cc}
1, & k \in R \\
0, & \text { otherwise. }
\end{array}\right.
\end{array}
$$

The distortion in the constellation cells will be decreased when the first configuration of the filter is repeated. However, the receiver can estimate the occurred clipping and compensate the received OFDM symbol accordingly because the clipping noise is generated by a known process mentioned in [11].

Finally, the F-TR signal can be converted into the time domain as follows:

$$
\begin{aligned}
\tilde{\mathbf{x}}(n) & =x(n)+\beta c(n) \\
& =x(n)+\beta \mathbf{F}_{q} \mathbf{C} .
\end{aligned}
$$

The structure of F-TR were given in Figure 2.

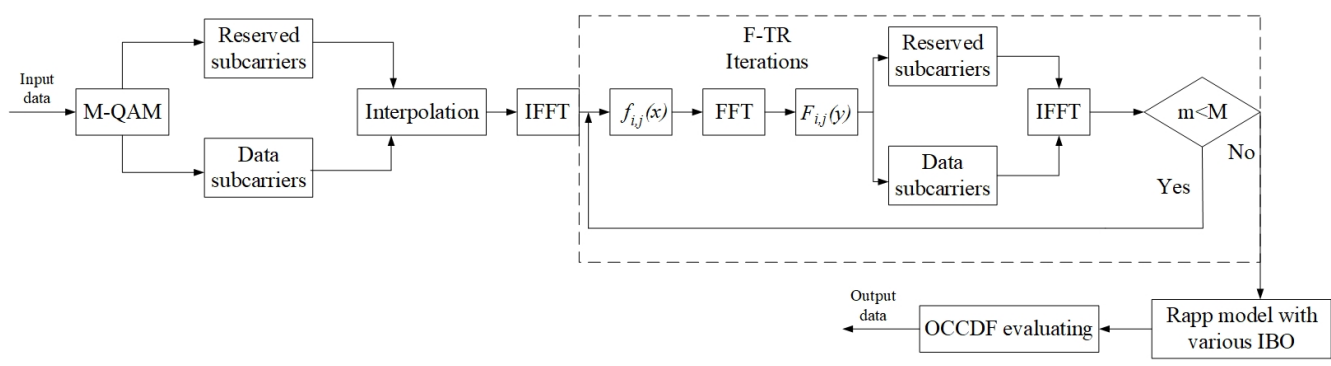

Figure 2. The structure of F-TR scheme.

In this paper, the increasing power of the F-TR symbol is irrelevant to MER but significantly affects IBO's implementation. To avoid taking the additive mentioned above power into account, the IBO definition can be adapted into:

$$
\mathrm{IBO}_{n}=P_{i n, \max }-P_{i n, a v}-P_{T R},
$$

where $P_{i n, a v}$ is the average input power, $P_{i n, \max }$ is the saturation point of an ideal amplifier (Rapp factor is infinite) for the input power, and $P_{T R}$ is the additive power with TR PAPR function. To describe the IBO gain more intuitively, $P_{T R}$ is subtracted from IBO gain to avoid taking into account F-TR strategies. In this case, an optimization model hybrid 
F-TR scheme can be adopted to minimize IBO by setting smoothness factor $p=10$ and determining the appropriate scaling factor $\partial_{l}$, amplitude threshold Asat as follow:

$$
\begin{aligned}
& \min _{f, F} I B O(\widetilde{\mathbf{x}}) \\
& \text { subject to } \operatorname{MER}(\widetilde{\mathbf{x}}) \geq \varepsilon \mathrm{dB},
\end{aligned}
$$

where $\varepsilon$ is the MER threshold (in $\mathrm{dB}$ ). This paper intended to reduce PAPR to exceed the prefined threshold received MER from the F-TR scheme. (32) and IBO can be simplified as follow:

$$
\min _{f, F} I B O(\widetilde{\mathbf{x}})=\varepsilon \mathrm{dB}
$$

In this paper, we comprehensively analyzed the PAPR reduction signal level amplitude and its statistical quantity from the overall level value distribution of OFDM signal and its statistical quantity perspective, thoroughly considering the impact from each subcarrier. We proposed novel maximizing HPA efficiency evaluation criteria in this paper to evaluate the optimized OFDM signal distribution probability curve.

After introducing the little distortion with the MER criterion, a lesser IBO value would increase HPA efficiency dramatically, so reducing IBO with fixed MER was regarded as the evaluation criterion for optimizing HPA efficiency.

By adjusting the distribution of total subcarriers in the F-TR constraint space, the HPA would achieve optimal efficiency. In other words, minimized IBO would be captured by fixed MER and the appropriate setting of relevant parameters in the F-TR scheme. Maximizing HPA efficiency evaluation criteria can be shown as:

$$
\widetilde{X}_{n}^{\text {opt }}=\arg _{\widetilde{X}_{n}} \min \mathrm{IBO}_{n},
$$

where $\widetilde{X}_{n}^{\text {opt }}$ is the optimum distributed subcarriers achieved minimum IBO value.

\subsection{FPSO-TR}

We adopted the PSO algorithm to achieve the minimum IBO values by novel maximizing HPA efficiency evaluation criteria. The PSO algorithm search optimization parameter path achieves the group optimal goal by the cooperation between individuals [35]. Suppose that the position and velocity of the $i$-th particle are: $\mathbf{w}_{i}=\left\{w_{i 1}, w_{i 2}, \ldots, w_{i D}\right\}, \mathbf{v}_{i}=$ $\left\{v_{i 1}, v_{i 2}, \ldots, v_{i D}\right\}$ respectively, where $i=1,2, \ldots, n$. Particles are updated by adaptive function $\mathbf{f}_{i}=\left\{f_{i} \mid i=1,2, \ldots, P\right\}$ and velocity iteration function, the individual and population optimal location are updated as: $w_{i}^{p}$ best $=\left\{w_{i 1}^{p}\right.$ best,$w_{i 2}^{p}$ best $, \ldots, w_{i D}^{p}$ best $\} p_{i}$ best $=$ $\left\{p_{i 1}\right.$ best,$p_{i 2}$ best $, \ldots, p_{i D}$ best $\}$ and $w_{i}^{g}$ best $=\left\{w_{i 1}^{g}\right.$ best,$w_{i 2}^{g}$ best $, \ldots, w_{i D}^{g}$ best $\}$, respectively. In each iteration, the velocity and position are updated as the following rules:

$$
\begin{aligned}
& v_{i+1}=\omega_{i} \times v_{i}+c_{1} \times \text { rand } \times\left(w_{i}^{p} \text { best }-w_{i}\right)+c_{2} \times \text { rand } \times\left(w_{i}^{g} \text { best }-w_{i}\right) \\
& w_{i+1}=w_{i}+v_{i+1},
\end{aligned}
$$

where rand is random number ranging from $[0,25]$. The structure of the PSO algorithm is shown in Figure 3. 


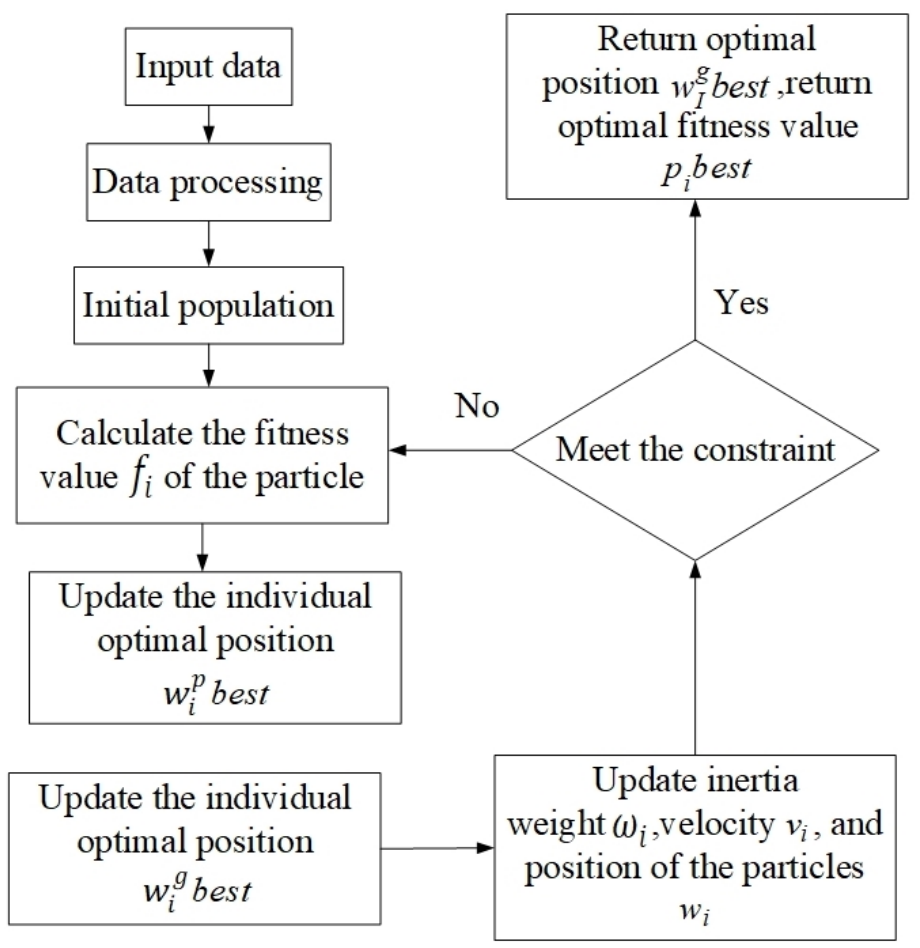

Figure 3. The structure of the PSO algorithm.

In this paper, F-TR iteration parameters are major parameters for predistortion adjustment, which determines the performance of MER and IBO. By setting fixed IBO, the optimal MER can be obtained by defined F-TR iteration parameters as population particles $\mathbf{w}_{i 1}=\left\{w_{11}, w_{21}, \ldots, w_{P 1}\right\}$ and $\mathbf{w}_{i 2}=\left\{w_{12}, w_{22}, \ldots, w_{P 2}\right\}$ respectively, the corresponding velocity defined as $\mathbf{v}_{i 1}=\left\{v_{11}, v_{21}, \ldots, v_{P 1}\right\}$ and $\mathbf{v}_{i 2}=\left\{v_{12}, v_{22}, \ldots, v_{P 2}\right\}$ respectively. The structure of the FPSO-TR algorithm is given in Figure 4.

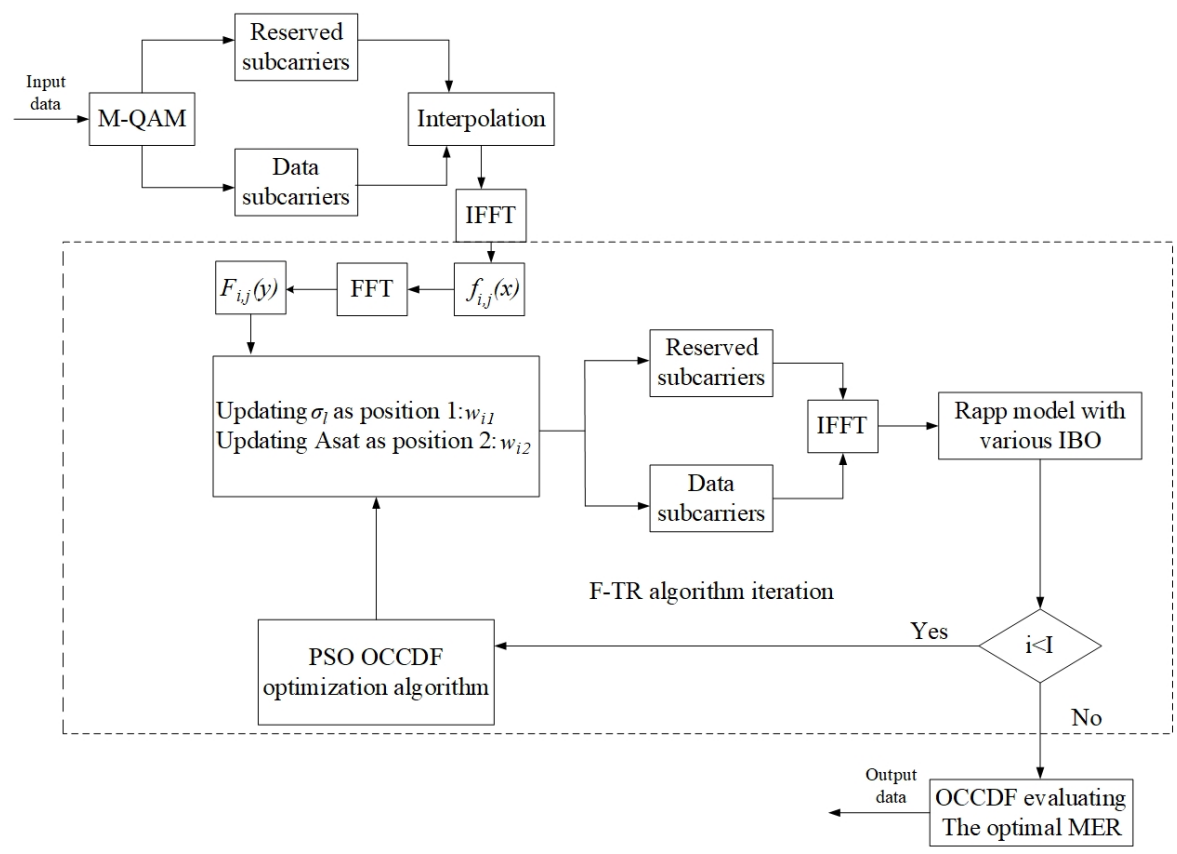

Figure 4. Structure of the FPSO-TR scheme. 
The FPSOPSO-TR Algorithm 1 is given in Table 1 in detail.

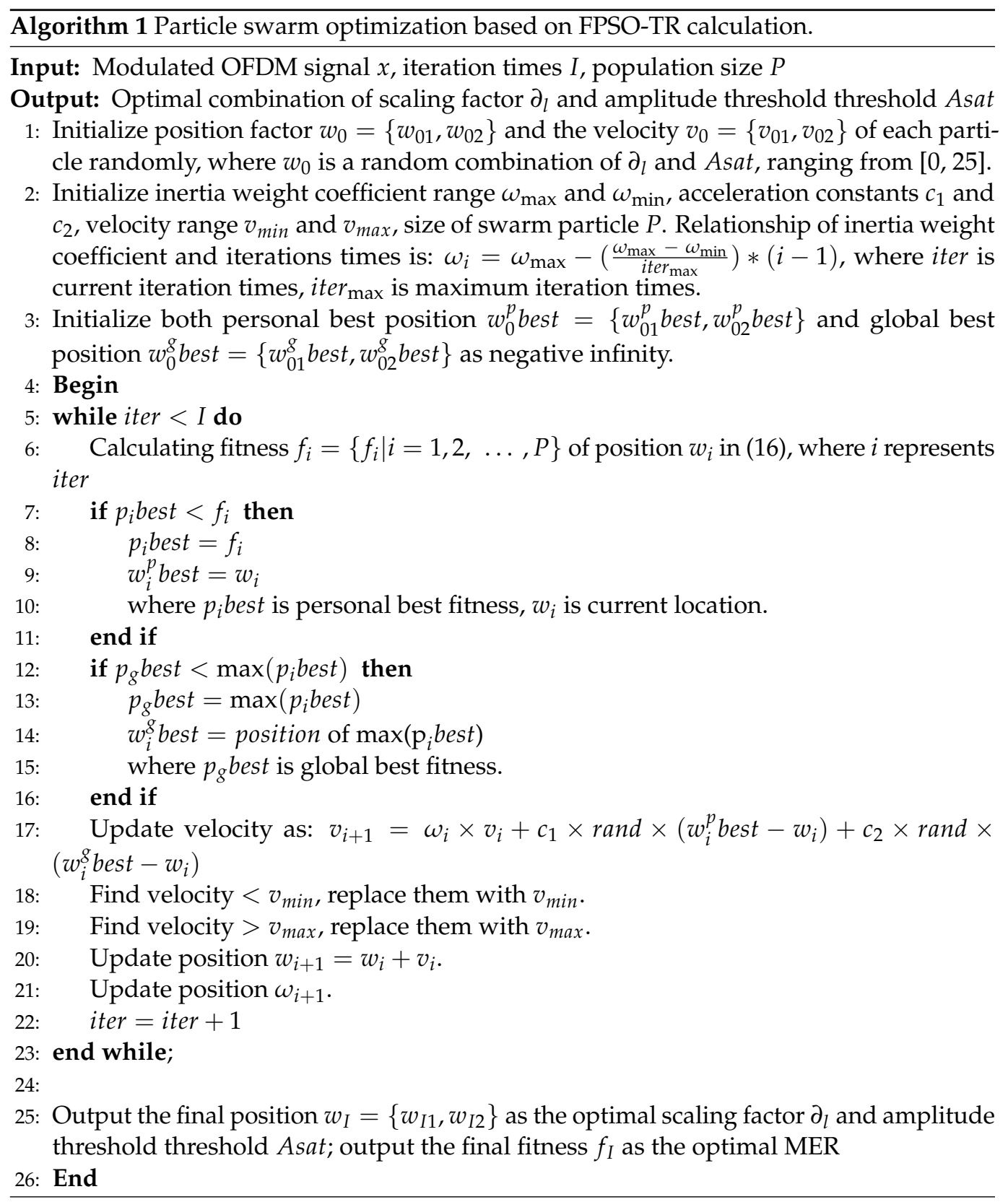

Table 1. Simulations parameters of Simulation results.

$\begin{array}{ll}\text { amplifier voltage gain } G & 1 \\ \text { Smoothing factor } p & 10 \\ \text { PAPR reduction scheme } & \text { F-TR } \\ \text { Total number of subcarrier } & 3780 \\ \text { Total number of OFDM symbols } & 10^{5} \\ \text { Pilot ratio } & 3 \%, 5 \%, 7 \% \\ \text { Up-sample times } & 4 \\ \text { Constellation modulation mode } & 16 \mathrm{QAM} \\ \text { PAPR threshold } & 6 \mathrm{~dB} \\ \text { Maximum iterations times } & 3\end{array}$




\section{Simulation}

To demonstrate the efficiency of the conventional TR scheme, this paper conducted a simulation that compared the PAPR reducing performance of PTS, ACE, and TR schemes with the OFDM system.

As shown in Figure 5, the original OFDM signal PAPR is almost $12 \mathrm{~dB}$ when the probability is $10^{-2}$, while the PAPR is $8.8 \mathrm{~dB}, 8.5 \mathrm{~dB}, 8.1 \mathrm{~dB}$ when the PAPR reduction scheme is ACE, PTS and TR respectively. Thus, TR has a significant PAPR reduction performance in the OFDM system. The conventional TR scheme PAPR reduction performance is more evident than when other methods are used.

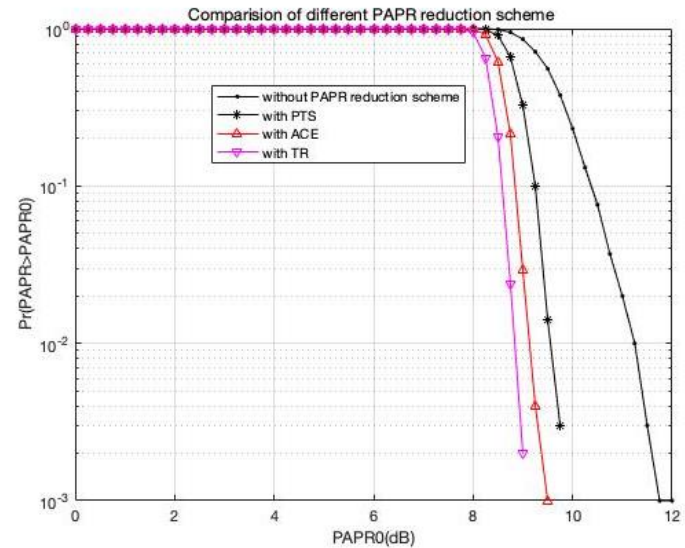

Figure 5. Comparison of different PAPR reduction scheme.

In this paper, performance simulations are shown for the proposed FPTS-TR. The OFDM signal modulated by 64QAM was adopted, 3780 subcarriers were generated. The original signal utilized four times upsampling. The Rapp HPA model employed $p=10$ in this paper (regardless of the nonlinear distortion of the amplifier). The parameters of FPTS-TR are given in Table 1.

To demonstrate the efficiency of the F-TR scheme with different pilot ratios on PAPR reduction performance, Figure 5 compares F-TR PAPR reduction performance when the pilot is $3 \%, 5 \%, 7 \%$, respectively.

As shown in Figure 6, the original OFDM signal PAPR is more than $11 \mathrm{~dB}$ when probability is $10^{-2}$, while the PAPR is $8.1 \mathrm{~dB}, 7.1 \mathrm{~dB}, 6.5 \mathrm{~dB}$ when the pilot ratio is $3 \%, 5 \%$, $7 \%$ respectively. Thus, F-TR has a significant PAPR reduction performance in the 64QAM OFDM system, and its performance enhanced with the increase of pilot ratio, 7\% pilot F-TR gained nearly $2 \mathrm{~dB}$ better than $3 \%$ pilot. However, CCDF standards failed to evaluate signal performance globally, and F-TR did not achieve optimal performance.

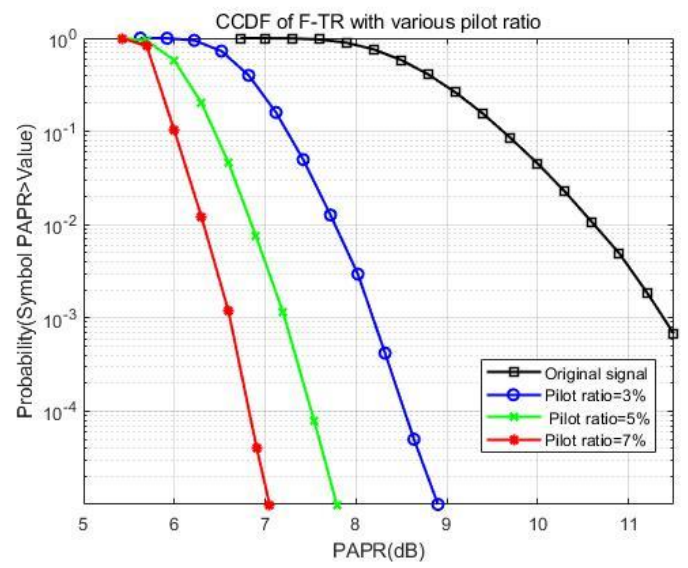

Figure 6. CCDF of F-TR with various pilot ratio. 
To achieve optimal MER performance, we adopted the FPSO-PTS scheme as in Figure 7 to study the relationship between pilot ratio and IBO gains, and Figure 7 shows the curve of the relationship mentioned above when $M E R=40 \mathrm{~dB}$; the PSO algorithm parameters setting are shown in Table 2.

It can be seen from Figure 7 that all curves have reached convergence and the optimal result has been searched; $3 \%$ pilot reached $M E R=41.03 \mathrm{~dB}$ by 22 times iterations; $5 \%$ pilot reached $M E R=44.27 \mathrm{~dB}$ by 14 times iterations; 3\% pilot reached $M E R=41.03 \mathrm{~dB}$ by 22 times iterations; $5 \%$ pilot reached $M E R=44.27 \mathrm{~dB}$ by 14 times iterations; $7 \%$ pilot reached $M E R=47.56 \mathrm{~dB}$ by 10 times iterations. Although $5 \%$ and $7 \%$ pilot are $3.24 \mathrm{~dB}$ higher than $3 \%$ pilot, $5 \%$ and $7 \%$ pilot had an almost identical performance. The FPTS-TR searching result is given in Table 3 in detail.

To evaluate the optimized OFDM signal distribution probability curve, Figure 8 adopted maximizing HPA efficiency evaluation criteria to evaluate the signal performance of F-TR after HPA, which employed the Rapp model when $p=10$.

Figure 8 shows that when $M E R=40 \mathrm{~dB}$, the IBO of the original signal is $7.95 \mathrm{~dB}$; After F-TR scheme with 3\% pilot, the IBO of the signal decreases by $6.9 \mathrm{~dB}$ and the gain is $1.05 \mathrm{~dB}$; After F-TR scheme with 5\% pilot, the IBO of the signal decreases by $6.6 \mathrm{~dB}$ and the gain is $0.3 \mathrm{~dB}$; After F-TR scheme with $7 \%$ pilot, the IBO of the signal decreases by $6.2 \mathrm{~dB}$ and the gain is $0.35 \mathrm{~dB}$. Thus, the IBO of F-TR can achieve more gains than the original 64QAM OFDM signal. In a specific range, the IBO gain enhanced with the increase of pilot ratio. It is clear that novel maximizing HPA efficiency evaluation criteria cannot clarify the HPA efficiency.

Table 2. FPSO-TR parameters setting.

\begin{tabular}{cc}
\hline PAPR reduction scheme & FPSO-TR \\
IBO & $7 \mathrm{~dB}$ \\
Searching positions (D) & 2 \\
Population size & 50 \\
Iteration times & 50 \\
Dimension values & 1 \\
Position ranges & {$[0,25]$} \\
$v_{\min }$ & -1 \\
$v_{\max }$ & 1 \\
$\omega_{\min }$ & 0.4 \\
$\omega_{\max }$ & 0.9 \\
$c_{1}$ & 0.5 \\
$c_{2}$ & 0.5 \\
\hline
\end{tabular}

Table 3. Results of FPTSO-TR.

\begin{tabular}{ccc}
\hline & Optimal $\partial_{l}$ & 23.17 \\
Pilot ratio $=3 \%$ & Optimal Asat & $14.45 \mathrm{~dB}$ \\
& Optimal MER & $41.03 \mathrm{~dB}$ \\
\hline \multirow{2}{*}{ Pilot ratio $=5 \%$} & Optimal $\partial_{l}$ & 22.91 \\
& Optimal Asat & $13.94 \mathrm{~dB}$ \\
& Optimal MER & $44.27 \mathrm{~dB}$ \\
\hline \multirow{2}{*}{ Pilot ratio $=7 \%$} & Optimal $\partial_{l}$ & 0.8 \\
& Optimal Asat & $13.7 \mathrm{~dB}$ \\
& Optimal MER & $47.56 \mathrm{~dB}$ \\
\hline
\end{tabular}




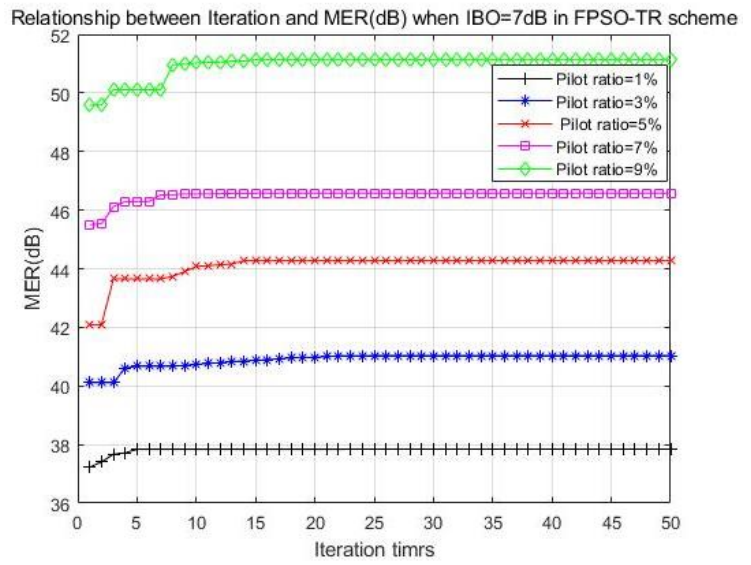

Figure 7. Relationship of IBO (dB) and pilot ratio (\%) in FPSO-TR scheme.

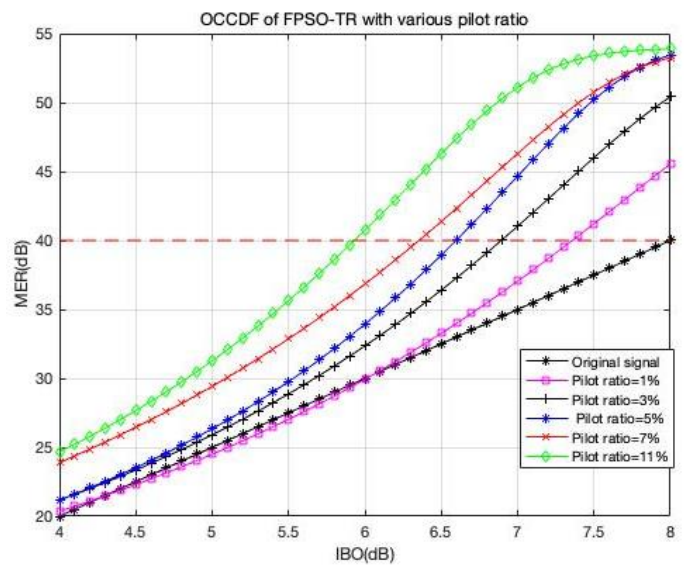

Figure 8. Novel maximizing HPA efficiency evaluation criteria.

It can be seen from Figure 9 that: the original signal IBO is $6 \mathrm{~dB}$ (pilotratio $=0$ ); IBO decreases with the increase of pilot ratio with trend slow down. IBO is $4.4 \mathrm{~dB}$, and the IBO gain is $1.6 \mathrm{~dB}$ when the pilot ratio reaches $20 \%$. It further proves the effectiveness of the F-TR algorithm.

By adopting the FPSO-TR scheme and maximizing HPA efficiency evaluation criteria, the best IBO and MER can be found and the efficiency of HPA would be improved.

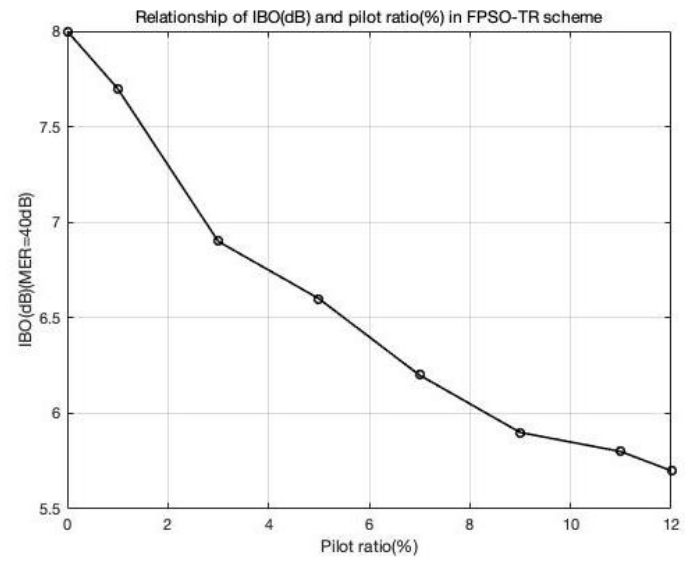

Figure 9. Relationship of IBO $(\mathrm{dB})$ and pilot ratio (\%) in F-TR scheme. 
Energy efficiency and signal to noise ratio (SNR) are demonstrated in Figure 10 and 11. Simulation parameters are shown in Table 4 . The energy efficiency of different pilot signals with $M E R=40 \mathrm{~dB}$ is fair compared.

It can be seen that the overall energy efficiency increases first and then decreases while SNR increases; the energy efficiency reaches the peak value between $S N R=[12,17] \mathrm{dB}$. The energy efficiency increases with the increases of the pilot when pilot $\leq 9 \%$. pilot $=1 \%$ and pilot $=9 \%$ reaches the minimum and maximum energy efficiency values of $10.9(\mathrm{~s} . \mathrm{Hz}) / \mathrm{J}$ and $16.5(\mathrm{~s} . \mathrm{Hz}) / \mathrm{J}$ respectively when $S N R=17 \mathrm{~dB}$. Then the energy efficiency decreased with the increases of the pilot when pilot $>9 \%$. pilot $=13 \%$ reaches $16.2(\mathrm{~s} . \mathrm{Hz}) / \mathrm{J}$ when $S N R=17 \mathrm{~dB}$. Obviously, energy efficiency peaked at pilot $=9 \%$ when $S N R=17 \mathrm{~dB}$.

Table 4. Simulations parameters of the Rapp HPA model.

$\begin{array}{ll}\text { Transmit antennas TX }=\ell & 64 \\ \text { Receive antennas RX } & 64 \\ \text { Range of SNR } & 1 \sim 31 \mathrm{~dB} \\ \text { Circuit power consumption } \ell \cdot P_{\text {cir }} & 0.01 \\ \text { Static power consumption } P_{\text {sta }} & 0.01 \\ \text { Piot ratio } \xi=\text { pilot } & 0.1 \sim 0.3 \\ \text { HPA efficiency } \eta_{a} & 0.35\end{array}$

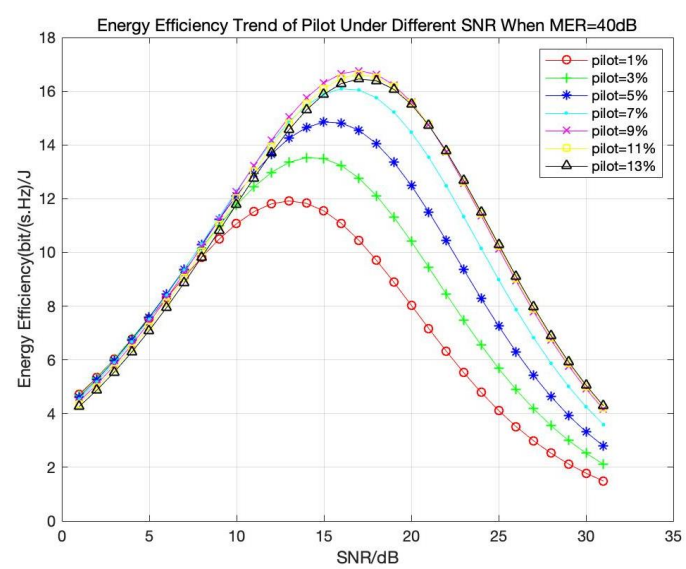

Figure 10. Energy Efficiency Trend of Pilot Under Different SNR When MER = $40 \mathrm{~dB}$.

Figure 11 demonstrates the relationship between pilot and energy efficiency when $S N R=17 \mathrm{~dB}$; the energy efficiency increases first and then decreases while SNR increases, peaking at pilot $=9 \%$ with the value of $16.5(\mathrm{~s} . \mathrm{Hz}) / \mathrm{J}$.

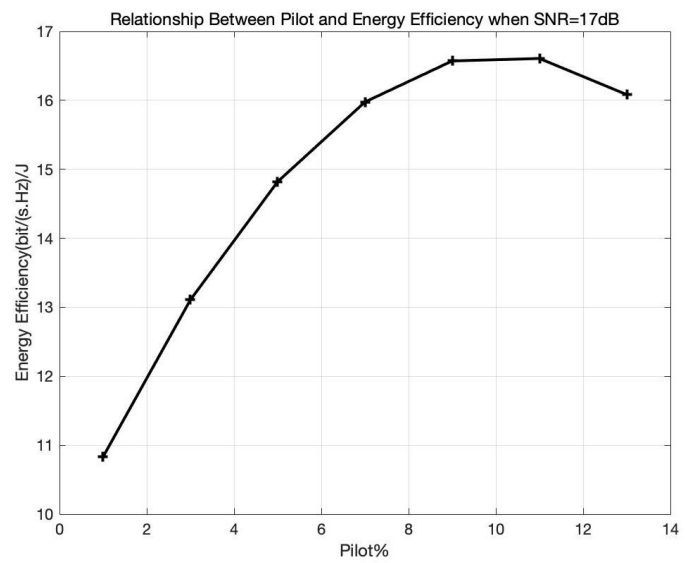

Figure 11. Relationship Between Pilot and Energy Efficiency when SNR $=17 \mathrm{~dB}$. 
This paper adopted an efficient PAPR reduction scheme TR to use HPA work in the linear region and saved energy for smart grids. The best MER results under different IBO conditions can be obtained by further combining FTR with the PSO algorithm, and the signal transmission accuracy of SG is improved. By the final simulation of energy efficiency, it can be concluded that the highest energy efficiency is obtained when $\mathrm{IBO}=9 \mathrm{~dB}$, which further enhances the energy efficiency of SG.

\title{
6. Conclusions
}

This paper studies the combination of the improved frequency domain TR method and the PSO algorithm and the novel maximizing HPA efficiency evaluation criteria of global evaluation to optimize the PAPR reduction performance and effect of FPTS-PSO high-order communication system in SGs. We adopted 3\%, 5\% and 7\% reserved tone in the frequency domain. The IBO achieved satisfactory gain compared to the original OFDM signal, which offset the power loss of HPA and solved the PAPR issue of the multi-carrier transmitter under high-order M-QAM modulation. By combining F-TR with the PSO algorithm, the optimal pilot ratio and the optimal power amplifier efficiency is obtained. FPSO-TR applied in the high-order communication system in SGs are proven to be an energy efficiency algorithm. In further work, the compatibility of the TR method with neural network algorithm methods can be considered to explore a better PAPR suppression scheme for a future communication system in SGs.

\begin{abstract}
Author Contributions: Conceptualization, F.H., Y.L. and L.J.; methodology, F.H., Y.L. and Z.X.; software, G.Z., J.X. and J.L.; validation, F.H., Y.L. and L.J.; formal analysis, F.H., Y.L., J.L. and L.J.; investigation, G.Z., J.X. and J.L.; resources, F.H., J.L. and L.J.; data curation, F.H., Y.L.; writingoriginal draft preparation, F.H., Y.L.; writing-review and editing, F.H., Y.L. and L.J.; visualization, F.H., Y.L. and L.J.; supervision, F.H., J.L. and L.J.; project administration, F.H., J.L. and L.J.; funding acquisition, F.H., J.L. and L.J. All authors have read and agreed to the published version of the manuscript.
\end{abstract}

Funding: This work was supported by "the Fundamental Research Funds for the Central Universities".

Conflicts of Interest: The authors declare no conflict of interest. The funders had no role in the design of the study; in the collection, analyses, or interpretation of data; in the writing of the manuscript, or in the decision to publish the results.

\begin{tabular}{|c|c|}
\hline \\
\hline \multicolumn{2}{|c|}{$\begin{array}{l}\text { Abbreviations } \\
\text { The following abbreviations are used in this manuscript: }\end{array}$} \\
\hline PAPR & Peak-to-Average Power Ratio \\
\hline OFDM & Orthogonal Frequency Division Multiplexing \\
\hline TR & Tone Reservation \\
\hline PSO & Particle Swarm Optimization \\
\hline IBO & Input Back-Off \\
\hline HPA & High Power Amplifier \\
\hline MER & Modulation Error Ratio \\
\hline EE & Energy Efficiency \\
\hline
\end{tabular}

\section{References}

1. Shaikh, M.H.N.; Bohara, V.A.; Aggarwal, P.; Srivastava, A. Energy Efficiency Evaluation for Downlink Full-Duplex Nonlinear MU-MIMO-OFDM System with Self-Energy Recycling. IEEE Syst. J. 2020, 14, 3313-3324. [CrossRef]

2. Liu, R.; Hai, X.; Du, S.; Zeng, L.; Bai, J.; Liu, J. Application of 5G network slicing technology in smart grid. In Proceedings of the 2021 IEEE 2nd International Conference on Big Data, Artificial Intelligence and Internet of Things Engineering (ICBAIE), Nanchang, China, 26-28 March 2021; pp. 740-743. [CrossRef]

3. Ye, G. Research on reducing energy consumption cost of 5G Base Station based on photovoltaic energy storage system. In Proceedings of the 2021 IEEE International Conference on Computer Science, Electronic Information Engineering and Intelligent Control Technology (CEI), Fuzhou, China, 24-26 September 2021; pp. 480-484. [CrossRef] 
4. Mahmud, M.H.; Hossain, M.M.; Khan, A.A.; Ahmed, S.; Mahmud, M.A.; Islam, M.H. Performance Analysis of OFDM, W-OFDM and F-OFDM Under Rayleigh Fading Channel for 5G Wireless Communication. In Proceedings of the 2020 3rd International Conference on Intelligent Sustainable Systems (ICISS), Thoothukudi, India, 3-5 December 2020; pp. 1172-1177. [CrossRef]

5. Ren, J.; Wang, T.; Wang, C.; Li, D. Low-PAPR Superimposed Layered ACO-OFDM for VLC Systems. In Proceedings of the 2020 12th International Conference on Communication Software and Networks (ICCSN), Chongqing, China, 12-15 June 2020; pp. 178-181. [CrossRef]

6. Fidele, M.; Damien, H.; Eric, N. Effect of Window Size on PAPR Reduction in 4G LTE Network Using Peak Windowing Algorithm in Presence of Non-linear HPA. In Proceedings of the 2020 IEEE 5th International Conference on Signal and Image Processing (ICSIP), Nanjing, China, 23-25 October 2020; pp. 1128-1133. [CrossRef]

7. Munni, T.N.; Hossam-E-Haider, M. A Hybrid Method Based on SLM and Exponential Companding Technique to Reduce the Peak to Average Power Ratio (PAPR) in an OFDM System. In Proceedings of the 2021 International Conference on Automation, Control and Mechatronics for Industry 4.0 (ACMI), Rajshahi, Bangladesh, 8-9 July 2021; pp. 1-5. [CrossRef]

8. Liu, X.; Zhang, X.; Zhang, L.; Xiao, P.; Wei, J.; Zhang, H.; Leung, V.C. PAPR Reduction Using Iterative Clipping/Filtering and ADMM Approaches for OFDM-Based Mixed-Numerology Systems. IEEE Trans. Wirel. Commun. 2020, 19, 2586-2600. [CrossRef]

9. Zhang, S.-Y.; Shahrrava, B. A SLM Scheme for PAPR Reduction in Polar Coded OFDM-IM Systems without Using Side Information. IEEE Trans. Broadcast. 2021, 67, 463-472. [CrossRef]

10. Marsalek, R.; Fedra, Z. Reduced Complexity Adaptive Symbol Selection Method for OFDM PAPR Reduction. In Proceedings of the 7th Nordic Signal Processing Symposium-NORSIG 2006, Reykjavik, Iceland, 7-9 June 2006; pp. 286-289. [CrossRef]

11. Tu, Y.-P.; Wu, F.-H.; Huang, Y.-F. A Novel Turbo Scheme Combining PTS with Adaptive TR for PAPR Reduction in OFDM Systems In Proceedings of the 2020 International Symposium on Computer, Consumer and Control (IS3C), Taichung City, Taiwan, 13-16 November 2020; pp. 122-125. [CrossRef]

12. Mukunthan, P.; Rajalakshmi, K.; Tamilselvi, K.; Vinothini, N. Papr reduction based on PTS combined interleaving and analysis of BER using hadamard code in OFDM systems. In Proceedings of the 2016 International Conference on Communication and Signal Processing (ICCSP), Melmaruvathur, India, 6-8 April 2016; pp. 2304-2308. [CrossRef]

13. Hassan, M.E.; Crussière, M.; Hélard, J.-F.; Nasser, Y.; Bazzi, O. Theoretical Performance of the Gradient-Based Tone Reservation PAPR Reduction Algorithm. In Proceedings of the 2020 16th International Conference on Wireless and Mobile Computing, Networking and Communications (WiMob), Thessaloniki, Greece, 12-14 October 2020; pp. 1-6. [CrossRef]

14. Samayoa, Y.; Ostermann, J. Modified Active Constellation Extension Algorithm for PAPR Reduction in OFDM Systems. In Proceedings of the 2020 Wireless Telecommunications Symposium (WTS), Washington, DC, USA, 22-24 April 2020; pp. 1-5. [CrossRef]

15. Xing, Z.; Liu, K.; Huang, K.; Tang, B.; Liu, Y. Novel PAPR reduction scheme based on continuous nonlinear piecewise companding transform for OFDM systems. China Commun. 2020, 17, 177-192. [CrossRef]

16. Wang, H.; Wang, X.; Xu, L.; Du, W. Hybrid PAPR Reduction Scheme for FBMC/OQAM Systems Based on Multi Data Block PTS and TR Methods. IEEE Access 2016, 4, 4761-4768. [CrossRef]

17. Xiao, Y.; Zhang, L.; Imran, M. Active Constellation Extension for Peak Power Reduction Based on Positive and Negative Iterations in OFDM Systems. In Proceedings of the 2019 UK/ China Emerging Technologies (UCET), Glasgow, UK, 21-22 August 2019; pp. 1-5. [CrossRef]

18. Wu, C.; Lu, Y.; Liu, G. An Improved Clipping Scheme Based on TR for PAPR Reduction in OFDM Systems. In Proceedings of the 2012 IEEE 12th International Conference on Computer and Information Technology, Chengdu, China, 27-29 October 2012; pp. 598-601. [CrossRef]

19. Li, Z.; Jin, N.; Wang, X.; Wei, J. Extreme Learning Machine-Based Tone Reservation Scheme for OFDM Systems. IEEE Wirel. Commun. Lett. 2021, 10, 30-33. [CrossRef]

20. Li, L.; Tellambura, C.; Tang, X. Improved Tone Reservation Method Based on Deep Learning for PAPR Reduction in OFDM System. In Proceedings of the 2019 11th International Conference on Wireless Communications and Signal Processing (WCSP), Xi'an, China, 23-25 October 2019; pp. 1-6. [CrossRef]

21. Xiao, Y.; Lei, X.; Wen, Q.; Li, S. A Class of Low Complexity PTS Techniques for PAPR Reduction in OFDM Systems. IEEE Signal Process. Lett. 2007, 14, 680-683. [CrossRef]

22. Purushothaman, K.E.; Madhuvathani, V.; Nagarajan, V. Design and Simulation of OFDMA Transceiver for High Speed 5G Wireless Network using Immense PSO-GA. In Proceedings of the 2019 International Conference on Communication and Signal Processing (ICCSP), Chennai, India, 4-6 April 2019; pp. 975-979. [CrossRef]

23. Gomes, R.; Duarte, L.; Ribeiro, C.; Hammoudeh, A.; Caldeirinha, R.F.S. A novel MIMO-OFDM Alamouti architecture for 5G systems at $26 \mathrm{GHz}$. In Proceedings of the 2019 SBMO/IEEE MTT-S International Microwave and Optoelectronics Conference (IMOC), Aveiro, Portugal, 10-14 November 2019; pp. 1-3. [CrossRef]

24. Watanabe, I.; Nishimori, K.; Taniguchi, R.; Murakami, T. Effectiveness of the virtual massive MIMO in 5G with OFDM. In Proceedings of the 2020 International Symposium on Antennas and Propagation (ISAP), Osaka, Japan, 25-28 January 2021; pp. 211-212. [CrossRef]

25. Ramteke, S.B.; Deshmukh, A.Y.; Dekate, K.N. A Review on Design and Analysis of 5G Mobile Communication MIMO System with OFDM. In Proceedings of the 2018 Second International Conference on Electronics, Communication and Aerospace Technology (ICECA), Coimbatore, India, 29-31 March 2018; pp. 542-546. [CrossRef] 
26. Jia, W.; Wang, W.Q.; Cheng, J.; Hau, Y.; Kang, Z. Low PAPR OFDM-Chirp Modulation Signaling Scheme. In Proceedings of the 2021 IEEE International Geoscience and Remote Sensing Symposium IGARSS, Brussels, Belgium, 11-16 July 2021; pp. 7963-7966. [CrossRef]

27. Ramavath, S.; Kshetrimayum, R.S. Analytical calculations of CCDF for some common PAPR reduction techniques in OFDM systems. In Proceedings of the 2012 International Conference on Communications, Devices and Intelligent Systems (CODIS), Kolkata, India, 28-29 December 2012; pp. 393-396. [CrossRef]

28. Hei, Y.; Liu, X.; Li, W.; Wang, S.; Huo, M. Energy- and Spectral-Efficiency Tradeoff in Nonlinear OFDM System of Visible Light Communications. J. Lightwave Technol. 2021. [CrossRef]

29. Rapp, C. Effects of HPA-nonlinearity on 4-DPSK/OFDM-signal for a digital sound broadcasting system. In Proceedings of the Second European Conference of Satellite Communications, Liege, Belgium, 22-24 October 1991.

30. Le, N.P.; Safaei, F.; Tran, L.C. Antenna Selection Strategies for MIMO-OFDM Wireless Systems: An Energy Efficiency Perspective. IEEE Trans. Veh. Technol. 2016, 65, 2048-2062. [CrossRef]

31. Park, D.-h.; Song, H.-k. A New PAPR Reduction Technique of OFDM System with Nonlinear High Power Amplifier. IEEE Trans. Consum. Electron. 2007, 53, 327-332. [CrossRef]

32. Tekin, T.; Karakaya, B. SDR implementation of OFDM transmitter and receiver in case of time and frequency offset. In Proceedings of the 2018 26th Signal Processing and Communications Applications Conference (SIU), Izmir, Turkey, 2-5 May 2018; pp. 1-4. [CrossRef]

33. Jiang, T.; Li, C.; Ni, C. Effect of PAPR Reduction on Spectrum and Energy Efficiencies in OFDM Systems With Class-A HPA Over AWGN Channel. IEEE Trans. Broadcast. 2013, 59, 513-519. [CrossRef]

34. Wang, Y.; Chen, Z.; Gong, K. MER performance analysis of M-QAM-OFDM with Wiener phase noise. In Proceedings of the 2007 International Conference on Microwave and Millimeter Wave Technology, Guilin, China, 18-21 April 2007; pp. 1-4. [CrossRef]

35. Das, J.; Bansode, R. Performance evaluation of PAPR using PTS-PSO in MIMO-OFDM systems for various higher ordermodulation schemes. In Proceedings of the International Conference Workshop on Electronics Telecommunication Engineering (ICWET 2016), Mumbai, India, 26-27 February 2016; pp. 213-221. [CrossRef] 